\title{
Particle Filter for Context Sensitive Indoor Pedestrian Navigation
}

\author{
Pekka Peltola \\ Nottingham Geospatial Institute \\ Nottingham University, England \\ Email: pekka.peltola@nottingham.ac.uk
}

\author{
Chris Hill \\ Nottingham Geospatial Institute \\ Nottingham University, England \\ Email: chris.hill@nottingham.ac.uk
}

\author{
Terry Moore \\ Nottingham Geospatial Institute \\ Nottingham University, England \\ Email: terry.moore@nottingham.ac.uk
}

\begin{abstract}
Novel particle filter design combines foot mounted inertial (IMU), bluetooth low energy (BLE) and ultra-wideband (UWB) technologies along with map matching into a seamless integrated navigation system for indoors. The system was evaluated by 10 test walks along an $80 \mathrm{~m}$ long indoor track including stairs and running. $95 \%$ of the time the average error for a particle was below $3.1 \mathrm{~m}$ with filter completion success rate of 90\%. Furthermore, a system without UWB using only IMU, BLE and map matching achieved an average error for a particle to be below $3.6 \mathrm{~m}$ with filter completion success rate of $70 \%$.

The selected technologies and sensors are affordable and easily deployable. Inertial measurement unit's characteristics complement the disadvantages in the rf technologies and vice versa. The code for the rover can be implemented on a modern mobile device with a foot mounted imu.
\end{abstract}

\section{INTRODUCTION}

No single technology nor method for seamless indoor positioning yet exist. Multiple technologies have been previously studied. So far, an indoor positioning system of low energy consumption, high processing power and high accuracy has not been implemented.

There are advantages in using multiple technologies in deriving an accurate navigation solution. In an appropriately integrated sensor fusion filter the good sides of each technology can be used and the disadvantages discarded.

Context determination plays a significant role in filter parameter configuration. Deriving the correct context (walk, run, indoor, outdoor etc.) enables optimal filter settings for the current context and thereby helps in acquiring the best possible navigation solution.

In this paper, we discuss the obstacles in seamless indoor positioning and offer a tested solution in overcoming some of these obstacles.

\section{BACKGROUND}

Marie Curie project Multi-Pos works towards the goal of narrowing the gap between user requirements and positioning technology possibilities. A recent review comparison on current indoor positioning technologies indicates that bluetooth low energy (BLE) is very affordable indoor positioning technology [1]. Following BLE, timing based Ultra-wideband (UWB) technology option scores almost as much as ultrasound. The higher energy consumption on UWB chip reduces its applicability in modern mobile devices.
Prieto discusses in [2] on how to detect gait phases effectively using belief condensation. Recorded velocity, acceleration and angular velocity values are divided into clusters of stance and swing phases. Prieto divides the swing phase further into three more detectable stages. This enables more accurate context modeling rather than considering only the stance and the swing phases.

The challenge in tracking pedestrian movement with inertial sensors is the determination of the correct gait phase. Using zero updates the resulting drift in the IMU mechanisation process can be compensated. IMU can be located in the pocket or on foot and thus the resulting signals will be different. Interpretation of these signals that vary even more depending if the user is walking, crawling or running is a challenge. Adaptive measures are necessary in order to set up the zero update detection and mechanisation parameters correctly.

Bluetooth low energy beacons can be used for localisation by measuring received signal strengths. Faragher [3] examined BLE positioning using gaussian processes fingerprinting. As with wifi [4] the achievable accuracy is at best approximately 2 to 3 metres. The advantage with BLE is the easier control of transmission power, advertising rate and deployability.

Ultra-wideband timing based ranging accuracy is approximately $30 \mathrm{~cm}$ [5]. This depends on the environment, the amount of people and other obstacles. Decawave, the UWB ranging chip manufacturer, states that non line of sight reach is 35 metres. Further UWB characteristics can be checked in [6]. The high accuracy of UWB technology compensates for the higher power usage.

Cardinal heading aiding improves tracking performance especially in constrained corridor style areas [7]. Most common direction of movement in a particular area adjusts the navigation solution direction towards these cardinal headings. In [7] each particle weight was adjusted according to their heading. In open lobby areas heading aiding is feasible if furniture or other structures form areas that can restrict the heading.

Particle filter algorithm consists of three stages. These are propagation, update and resampling stages. When considering a pedestrian step and heading systems [8] propagation involves deriving new step length and heading from the imu data. This is then randomised by perturbing each particle according to the measurement uncertainties [7]. In the update stage, particles 
are compared with sensor measurements such as bluetooth or map wall locations and weighted accordingly. Weight is a measure of quality of the particle. Different weighting methods exist like weighting according to distance (or similar quantity) from the position measurement (or other relevant variable like RSS). Particle weight is increased if the new measurement was close. In the final stage, multinomial, systematic or residual resampling are available for different resampling needs [9]. Depending on the resampling strategy, particles are killed and regenerated or transformed. Degeneracy and too high resampling frequency can be tackled using Resample-Move and Block Sampling methods as discussed in [10].

Context for pedestrian systems can be defined by the style of movement (walk, crawl, run) [11]. Inertial sensors play a major role in this behavioural context definition. Another contextual viewpoint is the external effect like the spatial context. Adaptive classification by proximity was used in [12] for reducing the computational load in context determination.

\section{TRACKING SENSORS}

We built a positioning system using three sensor technologies and map matching. Each positioning system can function by itself within a limited area. But in order to have coverage for the whole selected floorplan an integrated solution is necessary. These subsystems are integrated using a particle filter. HP Stream x360 laptop running Debian recorded and timetagged the IMU, BLE and UWB subsystem data. Foot mounted inertial subsystem tracks on the floor level in 3D while BLE and UWB track the laptop at the height of approximately 1.4 metres and in 2D. The BLE and UWB anchors were placed at the height of 1.4 metres. The subsystems are fused using a particle filter producing a 2D solution. The lever arm between the IMU and RF measurements is not taken into account. The subsystems providing measurements for the particle filter are described next.

\section{A. Inertial Measurement Unit}

Lord Microstrain 3DM GX4-45 sensor is attached onto a left boot. C program records accelerometer and gyroscope readings at the rate of $62.5 \mathrm{~Hz}$. We mechanise these values to get the attitude and position changes of the foot. Zero velocity updates reduce the IMU velocity drift. In addition, a zero height constraint is used to reduce position drift by clamping the mechanisation result to ground at the end of each step except in the stairs. Zero velocity update detection implementation is a threshold value of the total acceleration vector magnitude. Pitch change magnitude threshold was used in the stairs.

Deadreckoning using IMU provides foot heading change, direction of movement change and position change measurements of each step to the particle filter. Heading of the foot and the direction of movement are different, although usually walk is forward motion. Update rate from IMU to the particle filter is thus stepwise. A measurement consists of a step start and stop time and the forementioned measurements (direction of movement and position change).

\section{B. Bluetooth Low Energy Anchors}

We used four Kontakt BLE beacons and two Raspberry Pi's with BLE capable adapters as anchors. The deployment is shown in Figure 1. Kontakt anchors send an advertisement signal to the environment every $100 \mathrm{~ms}$ using BLE channels 37,38 and 39. The power level was set to normal. Raspberry Pi's implementation advertises every 20ms using channel 37. Using only one channel has the benefit of smaller RSS value deviation [3]. Unfortunately Pi's adapter's power level is set to high and could not be adjusted. Due to being asynchronous the measurements race against each other in which one is to be measured next by the laptop. C program's scanning rate that was running on the laptop was set to scan every $20 \mathrm{~ms}$.

Lobby area, the three corridors and the stairs that are shown in Figure 1 were fingeprinted using signal strength values at 38 points. Gaussian processes regression radiomaps were constructed for each BLE anchor [3], [4]. Interpolated radiomaps have $10 \mathrm{~cm}$ grid size.

Averaged RSS measurement values of each anchor are fed to the BLE GP subsystem. Difference maps are constructed between the radiomaps and the measurement values for each anchor. After this the maps are superpositioned. The location with the resulting difference minimum and locations with RSS values that are up to $0.5 \mathrm{dBm}$ higher are picked. Average of these location values is sent to the particle filter as a measurement. In addition, the range of the picked location values in both $\mathrm{x}$ and $\mathrm{y}$ directions are sent to the particle filter a simple quality values of the measurement. This approach has a clear disadvantage if there are two separate peaks.

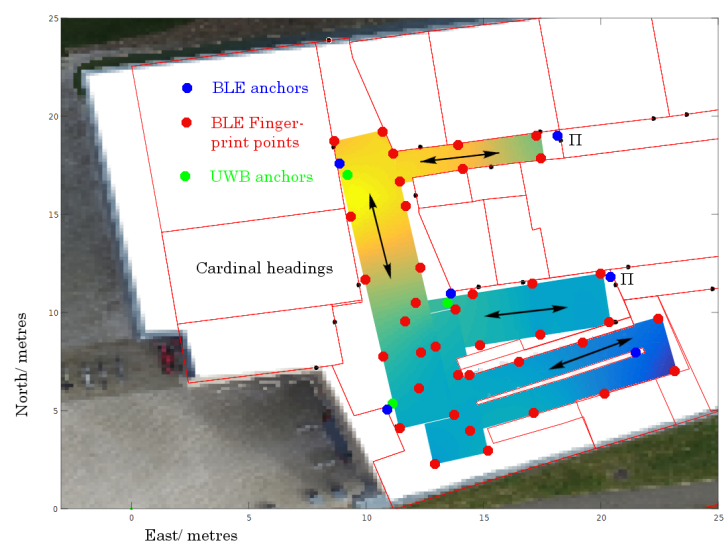

Fig. 1. BLE anchor positions (BLUE, Raspberry Pis are marked), BLE Fingerprinted area (RED) and UWB anchor positions (GREEN). Joint difference maps of the BLE anchor radiomaps and step measurement 7 of walk 3 is shown inside the fingerprinted area. Cardinal directions are shown in the corridors and stairs. No cardinal heading was applied in the lobby area.

\section{Ultra-Wideband Anchors}

Decawave TREK1000 kit was used to get UWB ranging measurements between the laptop and three anchors. The anchor positions are shown in Figure 1. C program for UWB 
recording provides timetagged range estimations every second. Triangulation is performed if all three anchors provide a ranging measurement. This gives a position estimate. Position error is estimated in both $\mathrm{x}$ and $\mathrm{y}$ directions by comparing the difference of the measured ranges and the ranges that the derived position solution has to the anchors.

\section{Map Matching}

Map contains information on wall locations. In this study we do not regard doors and leave the door opening and closing detection as a future research. All the doors are closed and act as walls in this study.

Second map matching feature is movement direction aiding. Each area on the map have principal movement directions as described previously (Figure 1). Map Matching step directs each particle's direction of movement stepwise for a few degrees (randomly between 0 to 5 degrees) towards the current closest principal direction.

\section{Contexts}

Context engine in this study is taken as a black box that works perfect. Context engine sets the correct filter settings for the current context. Figure 2 depicts this approach. Contexts included in this research are the following.

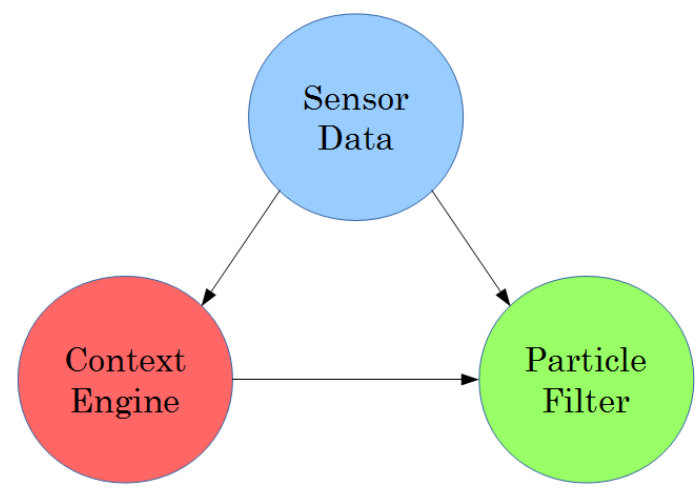

Fig. 2. The sensor data is fed to both Context Engine and Particle Filter. In this study we assume that the Context Engine works perfectly.

\section{A. Indoor}

Detecting whether a mobile device is indoors or outdoors is not as obvious for a device as it is for people. One possible method is to monitor the GNSS receiver for characteristics and clues for being located indoors as [13] proposes. BLE anchor placed on an entrance is as well a feasible method for indoor context detection.

\section{B. Stationary}

It is important to sense immobility in order to apply zero updates for the IMU mechanisation. While stationary, acceleration readings are not mechanised but gyroscope values are followed. This enables the tracking of the heading change, when the pedestrian shuffles feet at one spot or is immobile. When pedestrian moves again the direction of movement is tracked by mechanising both the acceleration and angular rate.

\section{Walk and Run}

Zero update detection filter has to adapt to the speed of the movement and thus to the magnitude of the recorded acceleration vectors. The time that a foot spends immobile is much shorter when running and zero update filter settings have to be set accordingly so that the mechanisation into a position works. Inertial measurement unit is in this respect the only sensor in this study that could provide the information to infer the correct context. Bluetooth and ultra-wideband technologies are still too inaccurate to measure such sudden inertial changes.

\section{Stairs}

Again the zero update detection settings are important in deriving the correct position change through the mechanisation process. Using just the pitch change was noticed to function better than the total acceleration vector for detecting zero updates in the staircase.

\section{Integration Using A PARTicle Filter}

We use a modified particle filter in MATLAB for sensor fusion. A particle consists of $\mathrm{x}$, $\mathrm{y}$ and floor level position information, direction of movement, step length and direction of movement change weights and of the previous values for positions and the direction of movement.

Figure 3 depicts the algorithm and is discussed next.

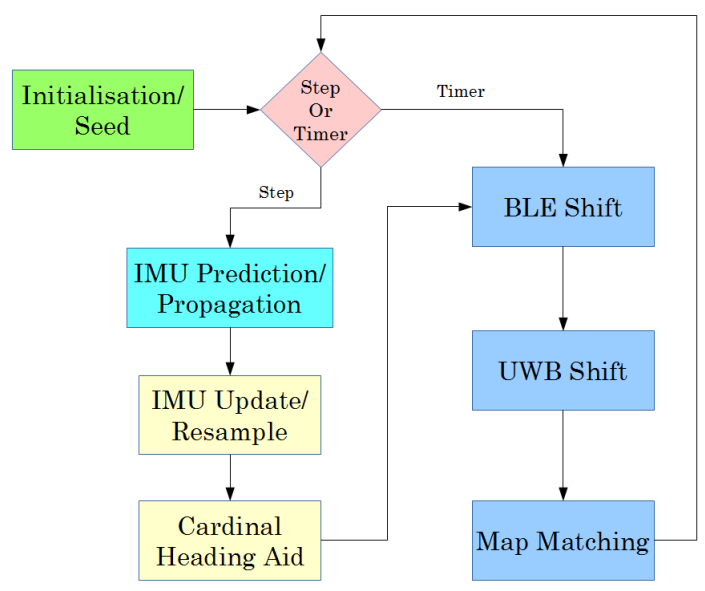

Fig. 3. Particle filter algorithm.

The mechanisation design enables inertial tracking with $100 \mathrm{~ms}$ delay. This is due to the zero update detection filter 8 tap delay. Rough mechanisation result can be provided with a 100ms delay and a fine mechanisation after a step has been completed. Measurements arrive either stepwise from the IMU or after a specified time period from the bluetooth or ultrawideband subsystems. If IMU outputs a step then the RF 
subsystems use the available measurements during midpoints of consequent steps. The particle filter design enables thereby a near real time processing with a delay of $1-2 \mathrm{~s}$ or in other words "a step behind solution". The designed particle filter consists of IMU propagation and update, cardinal heading, BLE and UWB shift and map matching phases.

1) IMU Prediction: IMU propagation phase predicts the next possible step location alike manner as the Kalman filter prediction step. For each particle a new direction of movement is predicted using the previous direction. New direction guess uses a wide normal distribution around the old direction. Circular mean is the previous direction and new direction of movement guesses are distributed so that the predictions cover a complete circle. Alternative prediction distributions for heading could be exploited here, but for simplicity this first version uses the circular mean from the previous direction. One very promising alternative is using learned previous track headings at the current position or goal points like corridors and doors. This would add somewhat more complicated prediction step where a comparison between previously learned most common tracking directions for current spatial location and the current location would be checked.

This approach enables multimodal heading tracking where particles can be distributed into two separate directions that are as probable. Figure 4 depicts this situation. A particle cluster's previous direction was changed into opposite direction. The IMU update weighs the particles according to the direction change and so two clusters of particles survive the IMU prediction and update phase.

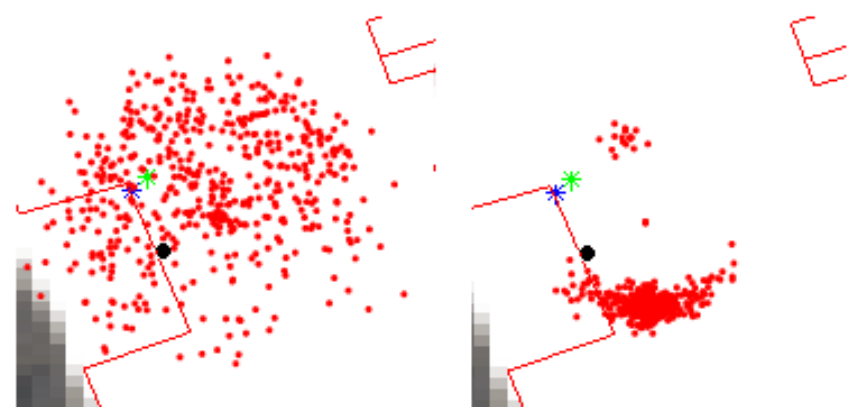

Fig. 4. Prediction is on the left. On the right are shown the particles after the IMU update. The previous direction of a bunch of particles were changed to simulate a multimodal direction prediction and thus survived the update weighing. Note that transformation is applied here (explained later in text).

The step length is estimated in a similar manner. Step length prediction distribution mean is at 1.5 metres and deviation towards greater values is much less than towards smaller values so that step length prediction is between 0 and 2 metres. Also values slightly below 0 and above 2 metres are possible but are not probable. This can be seen in the Figure 4. This can be adjusted and is useful for the running context. Predictions close to the measured step length survive.

2) IMU Update: Update and resampling phase compares the predicted new position of each particle with the IMU measurement values. Each particle's step length (position change) is compared with the mechanised measurement value and a step length weight is set according to how far it is from the measured value. Weighing scheme sets a new weight value between -1 and 1 using a normal distribution that converts the particle step length difference from the measured distance into a weight value. This distance to weight relation can be adjusted. Similar weighting method is used for the change in the direction of movement. Particles' step length weight that is over 0 survive the update process. Similarly particles' direction of movement change weight above 0 survive. Indexes of survived step lengths and directions are saved.

Particles position prediction with step lengths weights below 0 are transformed in the resampling phase. Using the survived step length indexes a new position is picked randomly from the survivor list for the particles that did not survive. This transforms the particle location into a location that did survive the comparison process. Similar method is used for direction of movement. Particle list is evaluated twice. In the end the weights are set back to zero. Particles are thus equal in quality after this phase. Further adjustments for the weighing design has been planned in order to include a measure for the quality of the each particle's history. This could be in the first place a flag or a counter that indicates how many times the particle has been transformed. This scheme would then indicate the most probable true track with a particle with the least transformations. Furthermore a combined track of two good quality particles at different time periods would likely represent the true trajectory.

3) Cardinal Headings: Cardinal heading aiding is applied after IMU measurement update. If the particle was not in the lobby a random direction change between 0 and 5 degrees is added to each particle's direction. The direction change is towards the cardinal directions that can be seen in the Figure 1.

4) BLE and UWB Shifts: The RF technology measurement integration inside the particle filter does not use a weighing scheme. Instead a shift of particle is introduced that depends on the quality of the measurement and of the distance between the particle and the measurement positions. Particles' locations are shifted toward the measurement value, which is the minimum of the BLE difference maps. The shift amount depends on the quality measure of the RF measurement.

In this first design two quality measures were used. BLE measurement quality is expressed by the deviation range in $\mathrm{x}$ and $y$ directions of the measurement value area. As explained previously the superposed RSS difference value locations that are $0.5 \mathrm{dBm}$ from the minimum value were chosen. The quality of the measurement is expressed in $\mathrm{x}$ and $\mathrm{y}$ directions by taking the inverse of the chosen value ranges. The larger the deviation range the smaller the shift. The formula 1 is a quality estimation, where $\mathrm{d}$ is the distance vector between particle and the measurement value and range is the $\mathrm{x}$ or $\mathrm{y}$ deviation mapped into dividend value $(1-30)$.

$$
\text { shift }=\frac{1}{f(\text { range })} \cdot d
$$


Similar approach was used for the UWB measurement. But in this case the quality/ weight measure was taken from the difference between the derived measurement position distances to the anchors and the range measurement values between anchor and the tag. This resembles pseudoranging. More sophisticated measurement quality indicators are being researched.

The behavioural context detection use is explained in the evaluation section that follows. The location context determination is evaluated using the mean location value for the particle cloud. Four different location contexts were included in the particle filter with different filter settings. Lobby and the corridor north of the lobby was the first location context area. The north corridor, the south corridor and the stairs were the three other location context areas. Filter settings were reset when the mean of the particles entered these areas.

The positioning solution of the particle filter is derived as the mean of all of the particles positions. This is why the form of the particle cloud or cluster is important in this filter design. More development is necessary if multimodal direction scheme is to be applied. A particle cluster detection offers an alternative for future versions of the software. Particles that are close to each other form a cluster and thus several clusters can have simultaneous positioning solutions. Moreover parallel particle filters can be run simultaneously if the device has enough processing power. These are not yet implemented into the software.

5) Map Matching: Final filter phase is the map matching that transforms particles locations again in a random order into particle locations that survived the wall crossing check. Particles' positions that crossed the walls are transformed into positions that did not cross any walls.

\section{System EVALUATION}

We completed 10 test walks inside the first floor of Nottingham Geospatial Building. The reference track is shown in Figure 5 as the red track. The test person walked directly towards the tape marked locations. Test subject stood still for 10 seconds at the point $\mathrm{X}$ in the map. After coming down the stairs the test person ran until reaching the end point.

The Figure 6 shows the cumulative distribution of error for five different positioning system combinations. These are the IMU only input for the particle filter, the BLE subsystem accuracy alone, the UWB subsystem when available by itself, the IMU + BLE measurement input for the particle filter and $\mathrm{IMU}+\mathrm{BLE}+\mathrm{UWB}$ combination particle filter.

From the Figure 6 we see that the UWB subsystem provides the greatest accuracy when available. The solution was only available in the lobby area and the corridor north of the lobby because of the limited amount of anchors. While moving, the dynamic accuracy of UWB algorithm is worse than when the test person stands still. Indeed when the test person stood still for ten seconds in the lobby area at the position marked with $X$ the accuracy of the UWB subsystem reached $30 \mathrm{~cm}$. The update rate of the UWB subsystem was 1 second and resulted in that while running, only two or three measurements were

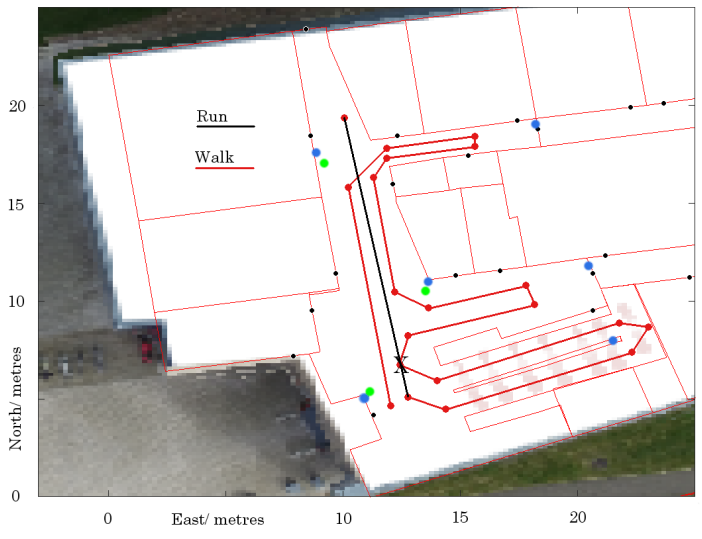

Fig. 5. The test walk track is marked in red colour. At point $\mathrm{X}$ the test person stood still for 10 seconds. Black track in the end is when the test person ran.

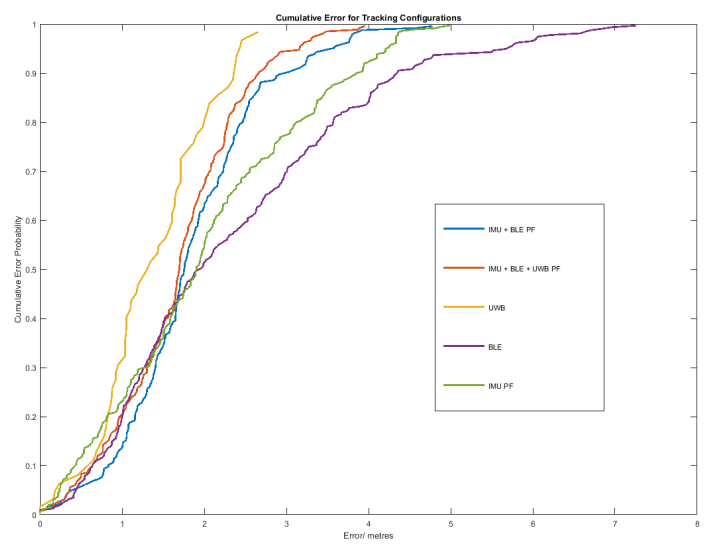

Fig. 6. UWB system is accurate when available. BLE availability is good but accuracy is the worst. Figure shows the errors when using IMU only, IMU + BLE and IMU + BLE + UWB measurement inputs for the particle filter.

available for the 16 metre indoor dash which also makes up the largest UWB errors in the error line.

The bluetooth low energy fingerprinting was expectedly the worst in terms of accuracy. At times, peaks 8 metres away from the true positions were recorded. It has to be noted here that sometimes the step detection picked a very short step, which results in very few BLE samples during this questionable step detection. BLE measurements were fed to the particle filter between midpoints of two consequent steps as presented before. Although low price and high measurement update rate compensate for this poor accuracy. Especially availability of a solution and easy deployability makes BLE a very promising alternative.

Similar BLE positioning behaviour as the Gaussian Fingeprinting was noticed in short tests conducted using an experimental pathloss modeling. The fingerprint map recording could become obsolete if this approach works well. This short 
test involves all line of sight anchors' RSS measurement values being converted into range measurements using a tabular RSS to distance conversion. Using similar shift approach for each particle they are either pushed or pulled towards the anchor depending on the converted distance values. This approach needs more testing.

IMU input alone for the particle filter resulted in 4 out of the 10 test walks completion percentage. Most often the particle filter failed when the particles advanced into the constrained staircase. Two clusters of particles divided into the staircase and back to the corridor. Staircase particles transformed into the corridor when they hit the narrow walls in the staircase. Filter failed when the particles bumped into the wall in the corridor, because it is shorter than the staircase. The poorer result for the IMU only particle filter can be seen in the Figure 6.

Bluetooth low energy solution helped with the staircase. The shift that is introduced each time the bluetooth update is run compresses the particle cloud towards one point. The particle cluster is thus more concentrated which prevented most of the particles in going into the south corridor. The standing before entering the stairs helped even more as the particle cluster concentrated in the lower end of the staircase. The completion rate was 7 out of 10 . The three failed walks were most likely caused by the the inaccurate starting location when entering the stairs. Slightly different mechanisation result might affect as well when going upstairs and going downstairs. Different approach for zero update detection for going upstairs and downstairs could be helpful. Filter failed again once in the stairs and twice when the particles bumped into the north wall of the lobby when running. One reason for bumping to the north lobby wall is that the cardinal heading aid was not applied in the lobby.

Finally the integration of the three IMU, BLE and UWB resulted in the best integrated navigation solution. Nine out of ten walks completed succesfully. This was most likely due to two reasons. UWB measurements while standing concentrated the particle cluster before entering the staircase. This in turn resulted in particles to be able to turn early enough before the final run.

\section{CONCLUSIONS}

More research is necessary in order to improve the performance and capabilities of the presented context sensitive particle filter. Controlling the prediction distributions is a promising way to achieve better positioning. Especially in constrained corridors like the stairs appropriate prediction settings are necessary in order for the filter not to fail. The mean of the particle cloud tends to move towards the center of the corridor as particles close to the wall are transformed. This has to be taken into account in the future version of the software.

As discussed previously, a track quality measure for each particle could help in prioritising the particles and picking the best quality tracks as the navigation solution instead of the cluster mean value. In open areas the particles tend to disperse which is why UWB shift works well in keeping the particle cluster more concentrated. Tracking multiple clusters simultaneously will add a backup solution if the main one turns out to be erroneous. Geometry of the building plan plays a big role with map matching techniques. Location based most common walking directions aid could help in guiding the particles towards the correct track.

UWB on a mobile device for positioning purposes is still coming slightly further in the future. IMU together with BLE is already very affordable configuration for commercial pedestrian navigation applications. The integrated seamless solution for indoor navigation is not too far ahead.

\section{ACKNOWLEDGEMENTS}

This work was financially supported by EU FP7 Marie Curie Initial Training Network MULTI-POS (Multi-technology Positioning Professionals) under grant nr. 316528.

\section{REFERENCES}

[1] Anahid Basiri, Pekka Peltola, Pedro Figueiredo e Silva, Elena Simona Lohan, Terry Moore, and Chris Hill. Indoor Positioning Technology Assessment using Analytic Hierarchy Process for Pedestrian Navigation Services. International Conference on Localization and GNSS (ICLGNSS), 2015

[2] Javier Prieto, Santiago Manuelas, and Moe Z. Win. Context-aided Inertial Navigation via Belief Condensation. IEEE Transactions on Signal Processing, 2016.

[3] Ramsey Faragher and Robert Harle. Location Fingerprinting With Bluetooth Low Energy Beacons. IEEE Journal on Selected Areas in Communications, 2015.

[4] Hao Jing, James Pinchin, Chris Hill, and Terry Moore. Wi-Fi Indoor Localisation based on Collaborative Ranging between Mobile Users. Proceedings of the 26th International Technical Meeting of The Satellite Division of the Institute of Navigation (ITM/ ION GNSS+), 2013.

[5] Francisco Zampella, Alessio De Angelis, Isaac Skog, Dave Zachariah, and Antonio Jimenez. A Constraint Approach for UWB and PDR Fusion. International Conference on Indoor Positioning and Indoor Navigation (IPIN), 2012.

[6] Xiaolin Meng, Yang Gao, King-Hung Kwok, and Hongbo Zhao. Assessment of UWB for ubiquitous positioning and navigation. Ubiquitous Positioning, Indoor Navigation, and Location Based Service (UPINLBS), 2012.

[7] James Pinchin, Chris Hide, and Terry Moore. A Particle Filter Approach to Indoor Navigation using a Foot Mounted Inertial Navigation System and Heuristic Heading Information. International Conference on Indoor Positioning and Indoor Navigation, 2012.

[8] Robert Harle. A Survey of Indoor Inertial Positioning Systems for Pedestrians. IEEE Communications Surveys \& Tutorials, 2013.

[9] James V. Candy. Bayesian Signal Processing: Classical, Modern and Particle Filtering Methods. Wiley, 2009.

[10] Arnaud Doucet and Adam M. Johansen. A Tutorial on Particle Filtering and Smoothing: Fifteen years later. Handbook of Nonlinear Filtering, Oxford University Press, 2009.

[11] Ulrich Walder and T. Bernoulli. Context-Adaptive Algorithms to Improve Indoor Positioning with Inertial Sensors. International Conference on Indoor Positioning and Indoor Navigation (IPIN), 2010.

[12] Sara Khalifa, Mahbub Hassan, and Aruna Seneviratne. Adaptive Pedestrian Activity Classification for Indoor Dead Reckoning Systems. International Conference on Indoor Positioning and Indoor Navigation (IPIN), 2013.

[13] Ondrej Daniel, Jussi Raasakka, Pekka Peltola, Markus Frhle, Alejandro Rivero-Rodriguez, Henk Wymeersch, and Jari Nurmi. Blind SubNyquist GNSS Signal Detection. 41st IEEE International Conference on Acoustics, Speech and Signal Processing (ICASSP), 2016. 